\title{
Trapping of Plasmons in Ion Holes
}

Padma Kant Shukla and Bengt Eliasson

Institut für Theoretische Physik IV, Fakultät für Physik und Astronomie, Ruhr-Universität Bochum, D-44780 Bochum, Germany

\section{Introduction}

We present analytical and numerical studies of a new electron plasma wave interaction mechanism which reveals trapping of Langmuir waves in ion holes associated with non-isothermal ion distribution functions [1]. This Langmuir-ion hole interaction is a unique kinetic phenomenon, which is governed by two second-order nonlinear differential equations in which the Langmuir wave electric field and ion hole potential are coupled in a complex fashion. Numerical analyses of our nonlinearly coupled differential equations exhibit trapping of localized Langmuir wave envelops in the ion hole which is either standing or moving slower than the ion thermal speed. The resulting ambipolar potential of the ion hole is essentially negative, giving rise to bipolar slow electric fields. The present investigation thus offers a new Langmuir wave contraction scenario that has not been explored in plasma physics before.

More than three decades ago, Hasegawa [2], Karpman [3, 4] and Zakharov [5] presented an elegant description of strong electromagnetic and Langmuir wave turbulence in which high-frequency photons and plasmons interact nonlinearly with low-frequency ionacoustic waves via the ponderomotive force arising due to the spatial gradient of the high-frequency wave intensity. This nonlinear interaction is typically described by the two-fluid and PoissonMaxwell equations, and the governing equations admit the localization of photon and plasmon wave packets, leading to the formation of envelope light and Langmuir wave solitons (also referred to cavitons) $[6,7,8,9]$. The latter are composed of electron/ion density depression which traps photon and Langmuir wave envelops. In two and three dimensions, one encounters photon self-focusing, Langmuir wave collapse $[5,10]$. The formation of cavitons has been observed in the ionosphere [11] as well as in several laboratory experiments $[12,13,14]$.

We present for the first time a new Langmuir turbulent state in the presence of ion phase-space vortices $[15,16,17]$ that are associated with density holes and bipolar electric fields in collisionless plasmas. Ion phase-space vortices are natural products of ion-beam driven two-stream instabilities, and they play a very important role in laboratory experiments $[18,19,20]$ as well as in the near-earth plasma environment [21, 22]. They are described by a wide class of Bernstein-Greene-Kruskal solutions to the Vlasov-Poisson equations. In the following, we show that nonlinearly coupled Langmuir waves and fully nonlinear ions holes admit a new class of solutions which have never been reported before. Specifically, we demonstrate the existence of standing and sub-ion thermal ion holes that trap Langmuir wave envelops.

\section{The mathematical model}

The ion hole in the presence of trapped Langmuir waves are governed by a coupled set of the nonlinear equations

$$
3 \frac{\partial^{2} W}{\partial \xi^{2}}-(\lambda-1) W-W \exp \left[\tau\left(\phi-W^{2}\right)\right]=0
$$

and

$$
\begin{aligned}
& \tau \frac{\partial^{2} \phi}{\partial \xi^{2}}-\exp \left[\tau\left(\phi-W^{2}\right)\right]+b \exp \left(-\frac{M^{2}}{2}\right) \times \\
& {\left[I\left(-\left(\phi-\phi_{\max }\right)\right)+K\left(\frac{M^{2}}{2},-\left(\phi-\phi_{\max }\right)\right)+\right.} \\
& \left.\frac{2}{\sqrt{\pi|\alpha|}} W_{D}\left(\sqrt{\alpha\left(\phi-\phi_{\max }\right)}\right)\right]=0,
\end{aligned}
$$

where $\phi$ is the ambipolar potential, $W$ is the Langmuir field envelope, $\lambda$ represents a nonlinear frequency shift, $\tau$ is the ion to electron temperature ratio, $\alpha$ is a trapping parameter for the trapped ions, $b$ is a normalization factor, and the Mach number $M$ is the ratio between the speed of the ion hole and the ion thermal velocity. The special functions $I, K$ and $W_{D}[15,1]$ result from the integration of the trapped and free ion distribution functions over velocity space.

\section{Numerical results}

We have carried out numerical studies of the equations governing ion holes with and without Langmuir waves for $\tau=0.1$ and $\alpha=-1.0$. We found that small-amplitude Langmuir waves can be linearly trapped in the density well of ion holes. Accordingly, for $W^{2} \ll 1$ Eq. (1) turns into a linear eigenvalue problem of the form

$$
3 \frac{d^{2} W}{d \xi^{2}}+[1-\exp (\phi)-\lambda] W=0,
$$

with the eigenvalue $\lambda$ and the corresponding eigen-function $W$, and where $\phi$ is obtained by assuming $W=0$ in the solution of Eq. (2); see the numerical solution of Eq. (2) in the form of ion density profiles and the associated ambipolar potentials in the left panels of Fig. 1

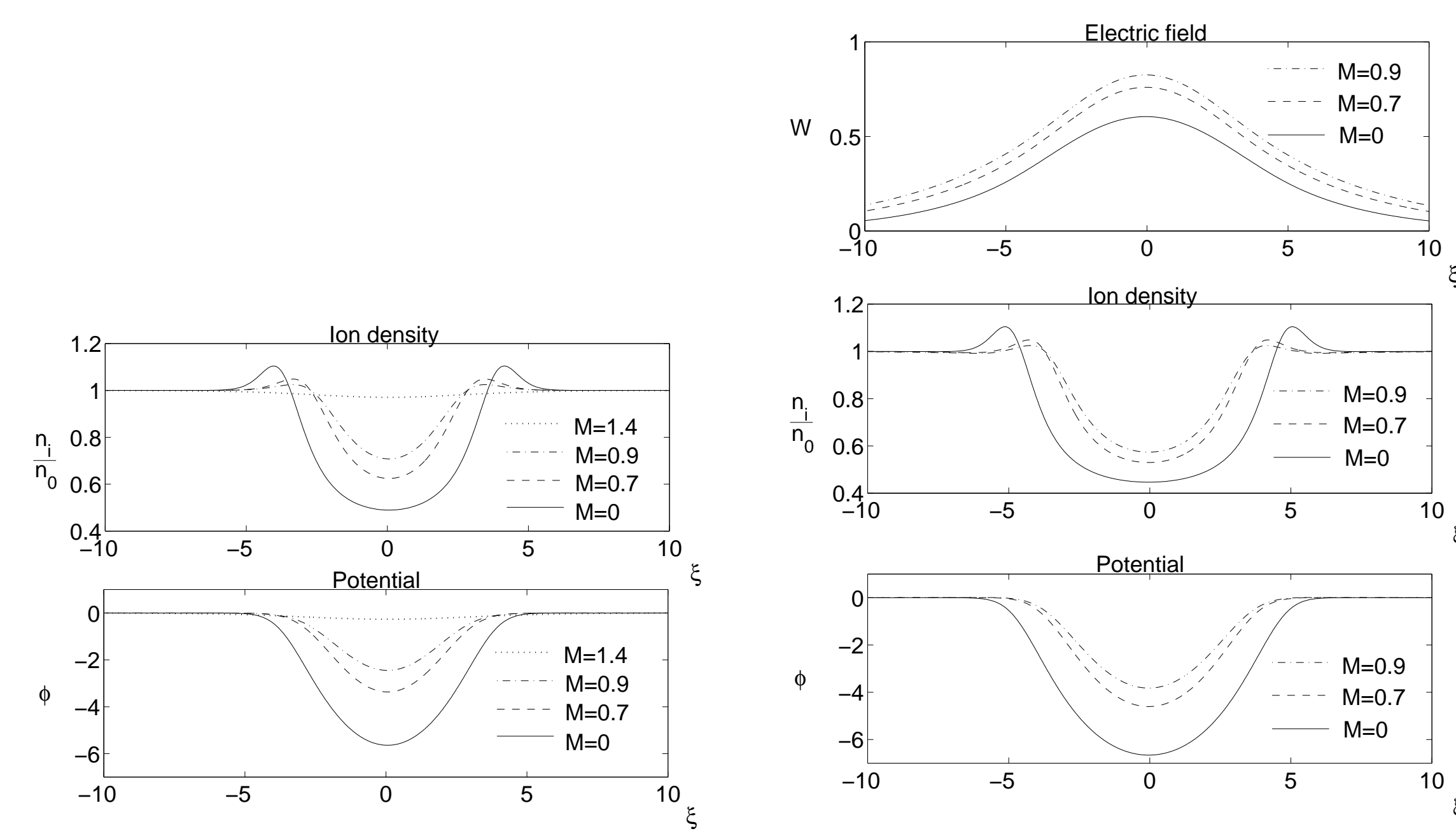

Figure 1. Ion holes without Langmuir waves ( $W=0$, left panel) and loaded with Langmuir waves (right panel) for different Mach numbers $M$, with $\tau=0.1$ and $\alpha=-1.0$.

The eigenvalue problem will have a continuous spectrum for $\lambda<0$, corresponding to "free particles" (in the language of quantum mechanics, ) and a point spectrum for $\lambda>0$, corresponding to "trapped particles." We have investigated numerically the cases corresponding to four different Mach numbers displayed in the left panels of Fig. 1, and found the corresponding positive eigenvalues listed in the second column of the table below

\begin{tabular}{l|l} 
Small amplitude & Finite amplitude
\end{tabular}

problem $\quad$ problem

$M=1.4 \lambda=0.0013 \quad M=1.4 \quad \lambda=0.1013$

$M=0.9 \lambda=0.0463 \quad M=0.9 \lambda=0.1463$

$M=0.7 \lambda=0.0772 \quad M=0.7 \quad \lambda=0.1772$

$M=0.0 \lambda=0.1906 \quad M=0.0 \lambda=0.2906$

where each eigenvalue $\lambda$ is associated to a bell-shaped eigenfunction $W$. Only one positive eigenvalue was found for each case, and thus these cases only admit the "ground states" for waves to be linearly trapped.

Next, we studied the presence of finite-amplitude Langmuir waves in the ion hole, in which the fully nonlinear system of equations (1) and (2) has to be solved numerically. The numerical solutions reveal that the ion hole deepened and widened, admitting the eigenvalue $\lambda$ to be larger. We investigated the special case with a nonlinear shift of 0.1 of $\lambda$ as listed in the fourth column in the table above, and found solutions for all cases except for $M=1.4$; the numerical solutions are depicted in the right panels of Fig. 1. We can see from Fig. 1 that the presence of trapped finite-amplitude Langmuir waves makes the ion density depletion both deeper and wider, and the same holds for the ambipolar potential well. The deepening of the ambipolar negative potential well is a feature closely related to the strongly non-isothermal trapped ion distribution function. For this case, the electrostatic potential had small-amplitude maxima $\phi_{\max }$ of the order $\approx 10^{-3}$ on each side of the ion hole, this maximum of the potential increased with increasing $M$.
It should be stressed that the properties of the present Langmuir

envelope solitons significantly differ from those based on Zakharov's model [5] which utilizes the fluid ion response for driven (by the Langmuir wave ponderomotive force) ion-acoustic perturbations and yield subsonic density depression accompanied with a positive localized ambipolar potential structure. Furthermore, consideration of a Boltzmann ion density distribution, viz. $n_{i}=n_{0} \exp (-\phi)$ would correspond to the case $M=0$ and $\alpha=1$ in Eq. (2). Here we have a localized Langmuir wave electric field envelope trapped in a standing ion density cavity. The corresponding slow ambipolar potential is positive and localized; see fig. 2 .

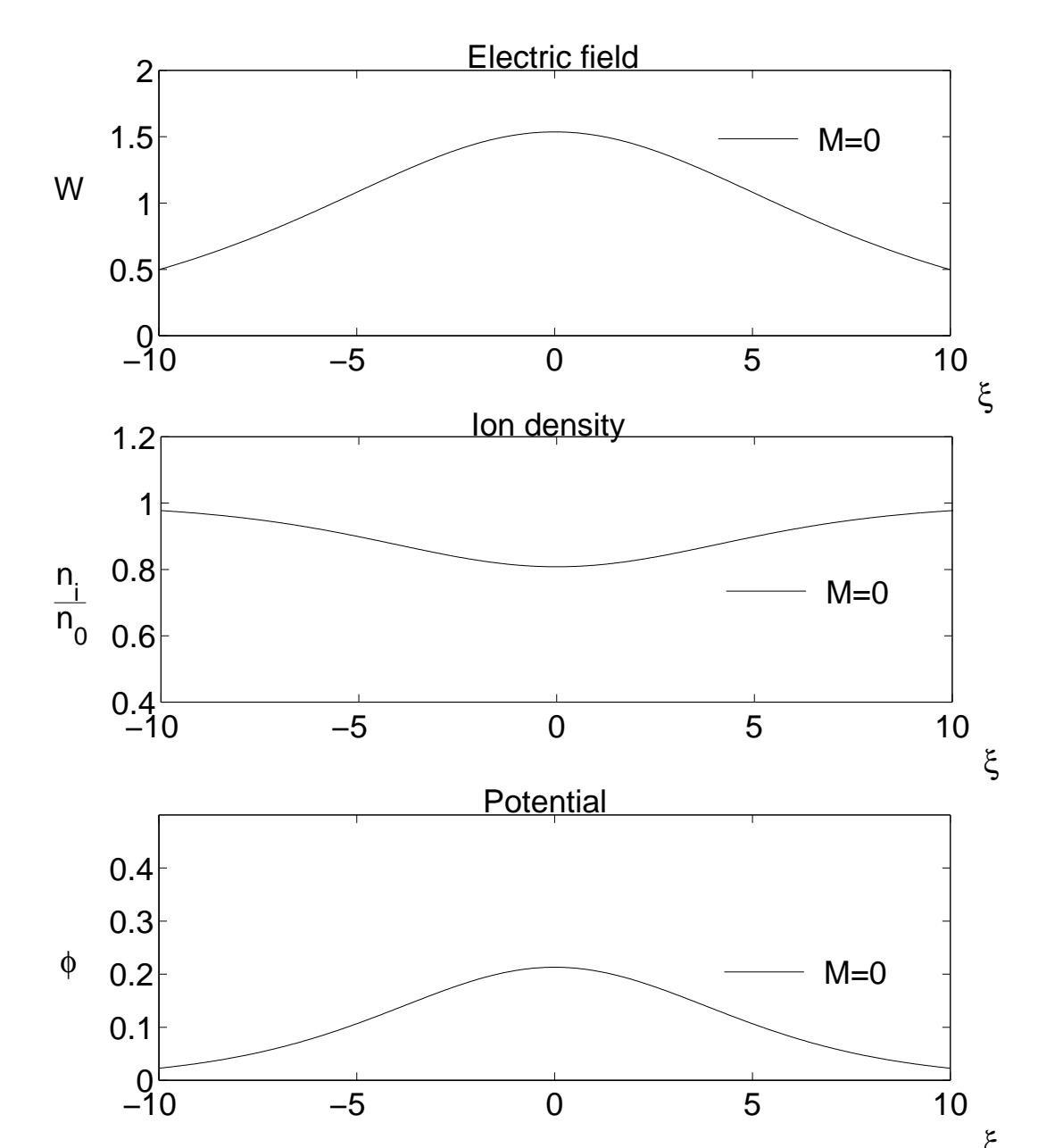

Figure 2. A Langmuir caviton with a Boltzmann ion distribution for $M=0$, $\lambda=0.1, \tau=0.1$ and $\alpha=1.0$.

Physically, the broadening of the ion hole and the enhancement of negative ambipolar potential occur because the ponderomotive force of the Langmuir waves locally expels electrons, which pull ions along to maintain the local charge neutrality. The deficit of ions in plasmas, in turn, produces more negative potential within the ion hole that is now widened and enlarged to trap the localized Langmuir wave electric field envelope. We have thus solved one of the fundamental problems of the nonlinear plasma physics which has potential applications in space and laboratory plasmas that are driven by electron and ion beams.

Reforences

[1] P. K. Shukla and B. Eliasson, JETP Lett., (Submitted, 2003).

[2] A. Hasegawa, Phys. Rev A 1, 1746 (1970).

[3] V. I. Karpman, Plasma Phys. 13, 477 (1971); Phys. Scr. 11, 263 (1975).

[4] V. I. Karpman, Nonlinear Waves in Dispersive Media (Pergamon, Oxford, 1975).

[5] V. E. Zakharov, Sov. Phys. JETP 35, 908 (1972).

[6] L. I. Rudakov, Dokl. Akad. Nauk SSSR 207 821 (1972) [Sov. Phys, Dokl. 17,1116 (1973).

[7] H. Schamel et al. Phys. Fluids 20, 1986 (1977); Deeskow et al, ibid. 30.2703 (1987).

[8] R. K. Varma and N. N. Rao, Phys. Lett. A 79, 311 (1980)

[9] N. N. Rao and R. K. Varma, J. Plasma Phys. 27,95 (1982).

[10] V. D. Shapiro and V. I. Sherchenko in Handbook of Plasma Physics Vol. II p. 8 (Nom Holland, Amsterdam, 1984); M. V. Goldman, Rev. Mod. Phys. 56. 709 (1984)

[111] H. C. Wong R. Stenzel, and A. Y Wong Phys. Rev Lett. 33886 (1974).

[12] A. Y. Wong and B. H. Quon, Phys. Rev. Lett. 34, 1499 (1975),

[13] H. Ikezi, R. P. H. Chang, and R. A. Stern, Phys. Rev. Lett. 36, 1047 (1976

[14] T. Intrator et al., Phys. Rev. Lett. 53, 1233 (1984).

[15] H. Schamel, Plasma Phys. 13. 491 (1971): Phvs. Reports 140, 161 (1986).

[16] S. Bujarbarua and H. Schamel, J. Plasma Physics 25, 515 (1981).

[17] F. Skiff et al., Phys. Plasmas 8, 3139 (2001).

[18] H. L. Pécseli et al., Phys. Lett. 81A, 386 (1981); Phys. Scripta 29, 241 (1984); H. L. Pécsel Laser Part. Beams 5, 211 (1987).

[19] G. Bachert et al., Phys. Rev. Lett. 80, 3260 (1998).

[20] Y Nakamura, H. Bailung and P. K. Shukla, Phys. Rey Lett 83 1602 (1999).

[21] C. Cattell et al., Geophys. Res. Lett. 29,9 (2002)

[22] P. Guio et al., Nonlinear Processes in Geophys. 10, 75 (2003).

23] R. Z. Sagdeev, in Reviews of Plasma Physics, edited by M. A. Leontovich (Consultants Bureau, New York, 1966), Vol. 4, p. 23; R. Z. Sagdeev, Rev. Mod. Phys. 51, 11 (1979). 